\title{
Pathological Behavior in the Spectral Statistics of the Asymmetric Rotor Model
}

\author{
V.R. Manfredi ${ }^{1}$ and L. Salasnich ${ }^{2}$ \\ ${ }^{1}$ Dipartimento di Fisica "G. Galilei", Università di Padova, \\ Istituto Nazionale di Fisica Nucleare, Sezione di Padova, \\ Via Marzolo 8, 35131 Padova, Italy, \\ ${ }^{2}$ Istituto Nazionale per la Fisica della Materia, Unità di Milano Università, \\ Dipartimento di Fisica, Università di Milano, \\ Via Celoria 16, 20133 Milano, Italy
}

\begin{abstract}
The aim of this work is to study the spectral statistics of the asymmetric rotor model (triaxial rigid rotator). The asymmetric top is classically integrable and, according to the Berry-Tabor theory, its spectral statistics should be Poissonian. Surprisingly, our numerical results show that the nearest neighbor spacing distribution $P(s)$ and the spectral rigidity $\Delta_{3}(L)$ do not follow Poisson statistics. In particular, $P(s)$ shows a sharp peak at $s=1$ while $\Delta_{3}(L)$ for small values of $L$ follows the Poissonian predictions and asymptotically it shows large fluctuations around its mean value. Finally, we analyze the information entropy, which shows a dissolution of quantum numbers by breaking the axial symmetry of the rigid rotator.

PACS numbers: 03.75.Fi, 05.30.Jp
\end{abstract}

Typeset using REVTEX 


\section{INTRODUCTION}

In the semiclassical limit $[1,2]$ there is a clear connection between the behavior of classical systems (regular or chaotic) and the corresponding quantal ones. For quantal systems, corresponding to classical regular systems, the spectral statistics $\left(P(s)\right.$ and $\left.\Delta_{3}(L)\right)$ follow the Poisson ensemble, while for systems corresponding to chaotic ones the Wigner ensemble is followed (see, for example, [3] and references therein).

Nevertheless, some exceptions are known. The most famous case is perhaps the harmonic oscillator one, discussed in great detail in references $[4,5]$. It has been also found that lowenergy spectral statistics of higher-dimensional separable Hamiltonian systems can show the level repulsion typical for chaotic systems. Especially critical in this sense are systems close to the harmonic oscillators and to rectangular wells [6].

The aim of this paper is to discuss another pathological case: the classically integrable triaxial rotator model (see, for instance, ref. [7]). Incidentally, this model has been used very often in the description of the low-lying states of the even-even atomic nuclei [8].

The asymmetric top described by the rotor model is a classically integrable system, but an analytical formula, as a function of quantum numbers, for its energy spectrum is not known. Nevertheless, numerical results can be obtained. By following the Landau approach [9], the Hamiltonian operator is split into 4 submatrices, corresponding to different symmetry

classes. Each truncated submatrix is numerically diagonalized. Finally, the nearest-neighbor spacing distribution $P(s)$ and the spectral rigidity $\Delta_{3}(L)$ are calculated. Surprisingly, the spectral statistics of energy levels do not follow the predictions of Poisson statistics.

\section{THE ASYMMETRIC ROTOR MODEL}

Let us consider a system of coordinates with axes along the three principal axes of intertia of the top, and rotating with it. The classical Hamiltonian $H$ of the top is given by

$$
\hat{H}=\frac{1}{2}\left(a J_{1}^{2}+b J_{2}^{2}+c J_{3}^{2}\right)
$$


where $\mathbf{J}=\left(J_{1}, J_{2}, J_{3}\right)$ is the angular momentum of the rotation and $a=1 / I_{1}, b=1 / I_{2}$, $c=1 / I_{3}$ are three parameters such that $I_{1}, I_{2}$ and $I_{3}$ are the principal momenta of intertia of the top. The Hamiltonian is classically integrable and its action variables are precisely the three components $J_{s}, s=1,2,3$, of the angular momentum [7].

The quantum Hamiltonian $\hat{H}$ is obtained by replacing the components of the angular momentum, in the classical expression of the energy, by the corresponding quantum operators $\hat{J}_{1}, \hat{J}_{2}$ and $\hat{J}_{3}$. The commutation rules for the operators of the angular momentum components in the rotating system of coordinates are given by

$$
\hat{J}_{r} \hat{J}_{s}-\hat{J}_{s} \hat{J}_{r}=-i \hbar \epsilon_{r s t} \hat{J}_{t}
$$

where $\epsilon_{r s t}$ is the Ricci tensor and $r, s, t=1,2,3$. Note that these commutation rules differ from those in the fixed system in the sign on the right-hand side [9].

As usual, the two operators $\hat{J}^{2}=\hat{J}_{1}^{2}+\hat{J}_{2}^{2}+\hat{J}_{3}^{2}$ and $\hat{J}_{3}$ are simultaneously diagonalized on the basis of eigenstates $|J, k\rangle$ with integer eigenvalues $J$ and $k(k=-J,-J+1, \ldots, J-1, J)$, respectively. The non-zero matrix elements of the quantum Hamiltonian $\hat{H}$ in the basis $|J, k\rangle$ are given by

$$
\begin{gathered}
\langle J, k|\hat{H}| J, k\rangle=\frac{\hbar^{2}}{4}(a+b)\left(J(J+1)-k^{2}\right)+\frac{\hbar^{2}}{2} c k^{2}, \\
\langle J, k|\hat{H}| J, k+2\rangle=\langle J, k+2|H| J, k\rangle= \\
=\frac{\hbar^{2}}{8}(a-b) \sqrt{(J-k)(J-k-1)(J+k+1)(J+k+2)} .
\end{gathered}
$$

The quantum Hamiltonian $\hat{H}$ has matrix elements only for transitions with $k \rightarrow k$ or $k \pm 2$. The absence of matrix elements for transitions between states with even and odd $k$ has the result that the matrix of degree $2 J+1$ is the direct product of two matrices of degrees $J$ and $J+1$. One of these contains matrix elements for transitions between states with even $k$, and the other contains those for transitions between states with odd $k$ [9].

It is useful to introduce a new basis, given by

$$
|J, k ; S\rangle=\frac{1}{\sqrt{2}}(|J, k\rangle+|J,-k\rangle), \quad|J, 0, S\rangle=|J, 0\rangle
$$




$$
|J, k ; A\rangle=\frac{1}{\sqrt{2}}(|J, k\rangle-|J,-k\rangle), \quad k \neq 0 .
$$

By using this new basis, the total Hamiltonian matrix is decomposed in the direct product of 4 submatrices by considering the parity of the quantum number $k$ : even (E) or odd (O), and the symmetry of the state: symmetric $(\mathrm{S})$ or anti-symmetric $(\mathrm{A})$. So the submatrices are labelled as follow: $(\mathrm{E}, \mathrm{S}),(\mathrm{E}, \mathrm{A}),(\mathrm{O}, \mathrm{S}),(\mathrm{O}, \mathrm{A})$. These are the classes of symmetry of the system. In Table 1 we show the dimension of each submatrix for a fixed $J$.

The matrix elements of the Hamiltonian $\hat{H}$ in the new basis, with respect to the old basis, are given by

$$
\begin{gathered}
\langle J, k, S|\hat{H}| J, k, S\rangle=\langle J, k, A|\hat{H}| J, k, A\rangle=\langle J, k|\hat{H}| J, k\rangle, \quad k \neq 1 \\
\langle J, 1, S|\hat{H}| J, 1, S\rangle=\langle J, 1|\hat{H}| J, 1\rangle+\langle J, 1|\hat{H}| J,-1\rangle \\
\langle J, 1, A|\hat{H}| J, 1, A\rangle=\langle J, 1|\hat{H}| J, 1\rangle-\langle J, 1|\hat{H}| J,-1\rangle \\
\langle J, k, S|\hat{H}| J, k+2, S\rangle=\langle J, k, A|\hat{H}| J, k+2, A\rangle=\langle J, k|\hat{H}| J, k+2\rangle, \quad k \neq 0 \\
\langle J, 0, S|\hat{H}| J, 2, S\rangle=\sqrt{2}\langle J, 0|\hat{H}| J, 2\rangle, \quad k \neq 0
\end{gathered}
$$

We calculate the eigenvalues of each submatrix for different values of $J$ using a fast implementation, in double precision, of the Lanczos algorithm with a LAPAC code [10]. In Figure 1 we plot the density of levels $\rho(E)$ of each submatrix of $\hat{H}$ and $J=1000$. The results show that the density of levels is practically the same for the four classes. $\rho(E)$ displays a high peak at the left-center of the energy interval and a long tail for large energy values.

\section{SPECTRAL STATISTICS}

As previously discussed, according to the Berry-Tabor theory [11,12], given a classical integrable Hamiltonian that, written in action variables $J_{r}$, satisfies the condition 


$$
\left|\frac{\partial^{2} H}{\partial J_{r} \partial J_{s}}\right| \neq 0
$$

then, in the semiclassical limit, its spectral statistics should follow the Poisson statistics. Note that a system of linear harmonic oscillators, whose Hamiltonian is given by $H=\omega \cdot \mathbf{I}$, does not satisfy the previous condition. In fact, a system of linear harmonic oscillators is integrable but it does not follow Poissonian statistics [4,5].

The triaxial rigid rotator is integrable and satisfies the Berry-Tabor condition (11). Thus, one expects that the spectral statistics of the quantized rigid rotator should be Poissonian. We shall show that is not the case.

In general, various statistics may be used to show the local correlations of the energy levels but the most used spectral statistics are $P(s)$ and $\Delta_{3}(L) . P(s)$ is the distribution of nearest-neighbor spacings $s_{i}=\left(\tilde{E}_{i+1}-\tilde{E}_{i}\right)$ of the unfolded levels $\tilde{E}_{i}$. It is obtained by accumulating the number of spacings that lie within the bin $(s, s+\Delta s)$ and then normalizing $P(s)$ to unit. As shown by Berry and Tabor [11,12], for quantum systems whose classical analogs are integrable, $P(s)$ is expected to follow the Poisson distribution

$$
P(s)=\exp (-s)
$$

The statistic $\Delta_{3}(L)$ is defined, for a fixed interval $(-L / 2, L / 2)$, as the least-square deviation of the staircase function $N(E)$ from the best straight line fitting it:

$$
\Delta_{3}(L)=\frac{1}{L} \min _{A, B} \int_{-L / 2}^{L / 2}[N(E)-A E-B]^{2} d E
$$

where $N(E)$ is the number of levels between $E$ and zero for positive energy, between $-E$ and zero for negative energy. The $\Delta_{3}(L)$ statistic provides a measure of the degree of rigidity of the spectrum: for a given interval L, the smaller $\Delta_{3}(L)$ is, the stronger is the rigidity, signifying the long-range correlations between levels. For this statistic the Poissonian prediction is

$$
\Delta_{3}(L)=\frac{L}{15}
$$


It is useful to remember that Berry, on the basis of the Gutwiller semiclassical formula for the density of states, has shown that $\Delta_{3}(L)$ deviates from the universal Poissonian predictions for large $L: \Delta_{3}(L)$ should saturate to an asymptotic value performing damped oscillations [13].

In Figure 2 the spectral statistic $P(s)$ is plotted for the four submatrices of $\hat{H}$ and $J=1000$. Note that the level spectrum is mapped into unfolded levels with quasi-uniform level density by using a standard procedure described in [14]. As expected from the previous analysis of density of levels, $P(s)$ is practically the same for the four classes of symmetry. Moreover, $P(s)$ has a pathological behavior: a peak near $s=1$ and nothing elsewhere. Compared to $P(s)$, the spectral rigidity $\Delta_{3}(L)$ is less pathological. As shown in Figure 3, $\Delta_{3}(L)$ follows quite well the Poisson prediction $\Delta_{3}(L)=L / 15$ for small $L$ but for larger values of $L$ it gets a constant mean value with fluctuations around this mean value. These fluctuations becomes very large by increasing $L$, in contrast with the Berry prediction [13].

The behavior of the density of levels $\rho(E)$ and of the spectral statistics $P(s)$ and $\Delta_{3}(L)$ does not change by changing the matrix dimension, namely the quantum number $J$. In Figure 4 we plot the density of levels and the spectral statistics for $J=2000$ and $J=4000$.

The results shown in the first four figures have been obtained with $a=1, b=\sqrt{2}$ and $\sqrt{5}$, in such a way that the rotor is triaxial. It is interesting to see what happens if one changes the deformation parameters $a, b$ and $c$, studying the transition from axial symmetry to triaxial symmetry. To do so, we take fixed $b$ and $c$ and modify $a$. In Figure 5 we plot the density of levels $\rho(E)$ for six values of $a$ ranging from $a=\sqrt{2}$ to $a=0$. The case $a=b=\sqrt{2}$ correspond to the axial symmetric one. The density of levels $\rho(E)$ is strongly modified by changing the parameter $a$, i.e. breaking the axial symmetry, but the spectral statistics are not, as shown by Figure 6 for the $P(s)$ distribution.

To conclude this section, we discuss another statistical quantity that has been proposed to study quantum chaos: the information entropy $S(E)$ of the eigenvector $\mid E>$ associated to the eigenvalue $E$ of the the Hamiltonian operator $\hat{H}[15]$. Given a generic basis set $\{\mid i>\}$, the eigenvector $\mid E>$ can be written as: 


$$
\left|E>=\sum_{i} c_{i}\right| i>
$$

where $c_{i}$ are the probability amplitudes. Then, the information entropy of the eigenvector $\mid E>$ with respect to the basis set $\{\mid i>\}$ is defined as

$$
S(E)=-\sum_{i}\left|c_{i}\right|^{2} \ln \left|c_{i}\right|^{2}
$$

The idea is that, just as in the classical theory a dissolution of integrability (with the KAM mechanism) simply means the onset of chaotic motion, in quantum systems a dissolution of quantum numbers may indicate the onset of quantum chaos (see also [16]). In figure 7 we show the information entropy $S(E)$ of the eigenvectors of the Hamiltonian matrix of

symmetry class $(\mathrm{E}, \mathrm{S})$ with respect to the axial symmetric basis set $\mid J, k, S>$, calculated for different values of the deformation parameter $a$. As expected, if the system has axial symmetry $(a=\sqrt{2})$ then the information entropy $S(E)$ is everywhere zero. By deforming the system, i.e. breaking the axial symmetry, $S(E)$ becomes positive and it is larger in the central part of the energy spectrum. It is important to stress that in our system the dissolution of quantum numbers shown in Figure 7 does not have a classical analog because the classical Hamiltonian is always integrable. Thus, in our case, a large $S(E)$ simply means a fully broken axial symmetry of the rigid rotor.

\section{CONCLUSIONS}

The main conclusion of this paper is that the asymmetric rotor is, like the harmonic oscillator, another pathological case with respect to the classical-quantum correspondence between integrability and Poisson statistics. In our opinion, the pathology of the asymmetric rotor model is more interesting because, unlike the harmonic oscillator, the asymmetric rotor satisfies the conditions of the Berry-Tabor theory. The presence of hidden symmetries could explain the pathological behavior of spectral statistics but such symmetries have not yet been identified. For the sake of completeness we remember that, as stressed by Rau [17], in classical mechanics, the asymmetric rotor and the nonlinear pendulum are intimately linked 
and form the basis for many studies of nonlinear dynamics. Finally, we have shown that the information entropy of eivenvectors with the respect to the axial symmetric basis set gives a clear signature of the breaking of axial symmetry of the rigid rotator, but the rigid rotator is always classically integrable.

V.R.M. is greately indebted to M.V. Berry and S. Graffi for enlightening discussions. L.S. acknowledges M. Robnik and J.M.G. Gomez for fruitful conversations. 


\section{TABLES}

\begin{tabular}{|c|c|c|c|c|}
\hline & $(E, S)$ & $E$, & $O$ & D, \\
\hline$J$ even & $\frac{J}{2}+1$ & $\frac{J}{2}$ & $\frac{J}{2}$ & $\frac{J}{2}$ \\
\hline$J$ odd & $\frac{J-1}{2}$ & $\frac{J+1}{2}$ & $\frac{J+}{2}$ & $\frac{J+1}{2}$ \\
\hline
\end{tabular}

Table 1. Number of states in each submatrix of the asymmetrical top Hamiltonian for a fixed $J$. 


\section{FIGURE CAPTIONS}

Figure 1: Density of levels $\rho(E)$ of the four classes of symmetry with $J=1000$. Parameters: $a=1, b=\sqrt{2}, c=\sqrt{5}$ and $\hbar=1$.

Figure 2: Nearest neighbor spacing distribution $P(s)$ of the four classes of symmetry with $J=1000$. The dashed line is the Poisson prediction $P(s)=\exp (-s)$. Parameters: $a=1, b=\sqrt{2}, c=\sqrt{5}$ and $\hbar=1$.

Figure 3: Spectral rigidity $\Delta_{3}(L)$ of the four classes of symmetry with $J=1000$. The dashed line is the Poisson prediction $\Delta_{3}(L)=L / 15$. Parameters: $a=1, b=\sqrt{2}, c=\sqrt{5}$ and $\hbar=1$.

Figure 4: Density of levels $\rho(E)$, Nearest neighbor spacing distribution $P(s)$ and spectral rigidity $\Delta_{3}(L)$ of the $(E, S)$ class of symmetry, with $J=2000$ (top) and $J=4000$ (bottom). Dashed lines are Poisson predictions. Parameters: $a=1, b=\sqrt{2}, c=\sqrt{5}$ and $\hbar=1$.

Figure 5: Density of levels $\rho(E)$ of the $(E, S)$ class of symmetry, with $J=1000$. Parameters: $b=\sqrt{2}, c=\sqrt{5}$ and $\hbar=1$. Different values of the deformation parameter: (a) $a=\sqrt{2}$, (b) $a=\sqrt{1.9}$, (c) $a=\sqrt{1.5}$, (d) $a=1$, (e) $a=\sqrt{0.5}$, (f) $a=0$.

Figure 6: Nearest neighbor spacing distribution $P(s)$ of the $(E, S)$ class of symmetry, with $J=1000$. Parameters: $b=\sqrt{2}, c=\sqrt{5}$ and $\hbar=1$. Different values of the deformation parameter: (a) $a=\sqrt{2}$, (b) $a=\sqrt{1.9}$, (c) $a=\sqrt{1.5}$, (d) $a=1$, (e) $a=\sqrt{0.5}$, (f) $a=0$.

Figure 7: Information entropy $S(E)$ of the $(E, S)$ class of symmetry, with $J=1000$. Parameters: $b=\sqrt{2}, c=\sqrt{5}$ and $\hbar=1$. Different values of the deformation parameter: (a) $a=\sqrt{2}$, (b) $a=\sqrt{1.9}$, (c) $a=\sqrt{1.5}$, (d) $a=1$, (e) $a=\sqrt{0.5}$, (f) $a=0$. 


\section{REFERENCES}

[1] V.P. Maslov and M.V. Fedoriuk, Semiclassical Approximation in Quantum Mechanics (Reidel Publishing Company, 1981).

[2] A.B. Migdal, Qualitative Methods in Quantum Theory (Benjamin, 1997).

[3] V.R. Manfredi and L. Salasnich, Int. J. Mod. Phys. B 13, 2343 (1999).

[4] A. Pandey, O. Bohigas and M.J. Giannoni: J. Phys. A: Math. Gen. 22, 4083 (1989).

[5] A. Pandey and R. Ramaswamy: Phys. Rev. A 43, 4237 (1991).

[6] S. Drozdz and J. Speth: Phys. Rev. Lett. 67, 529 (1991); 68, 3109 (1992).

[7] H. Goldstein, Classical Mechanics (Addision Wesley, Reading, 1980).

[8] J.M. Eisenberg and W. Greiner, Nuclear Models, vol. 1 (North Hollnd, Amsterdam, 1975).

[9] L. Landau and E. Lifshitz, Course in Theoretical Physics, vol. 3: Quantum Mechanics (Pergamon, 1977).

[10] LAPAC Fortran Library, Linear Algebra Package, NAG Ltd 2001.

[11] M.V. Berry and M. Tabor, Proc. Roy. Soc. Lond. A 356, 375 (1977); M.V. Berry, Annals of Phys. 131, 163 (1981).

[12] M. Tabor Chaos and Integrability in Nonlinear Dynamics (Wiley, New York, 1989).

[13] M. Berry, Proc. Roy. Soc. Lond. A 400, 229 (1985).

[14] V.R. Manfredi, Lett. Nuovo Cimento 40, 135 (1984).

[15] J. Reichl, Europhys. Lett. 6, 669 (1988).

[16] F. Sakata et al., Nucl. Phys. A 519, 93c (1990).

[17] A.R.P. Rau, Rev. Mod. Phys. 64, 623 (1992). 

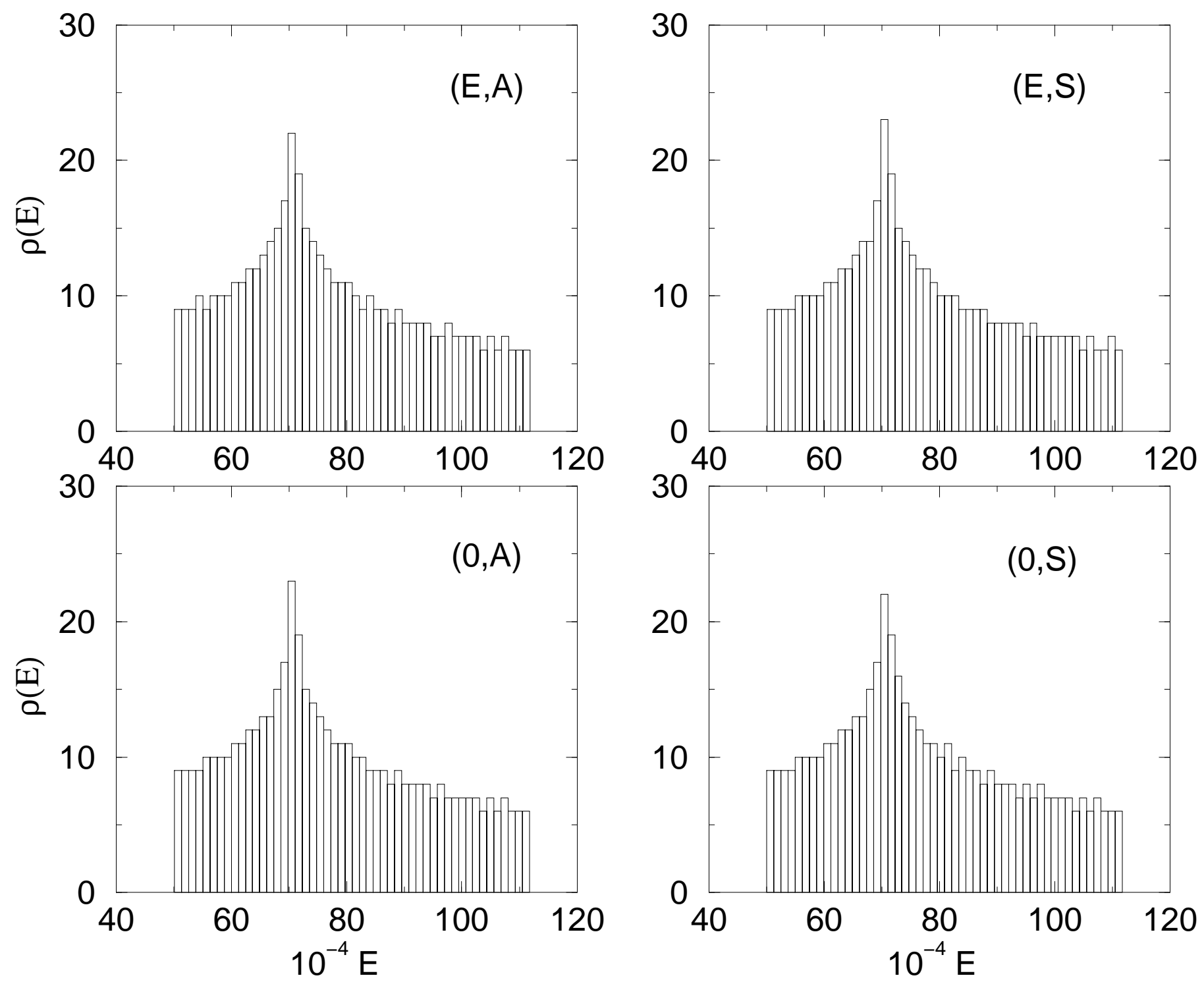

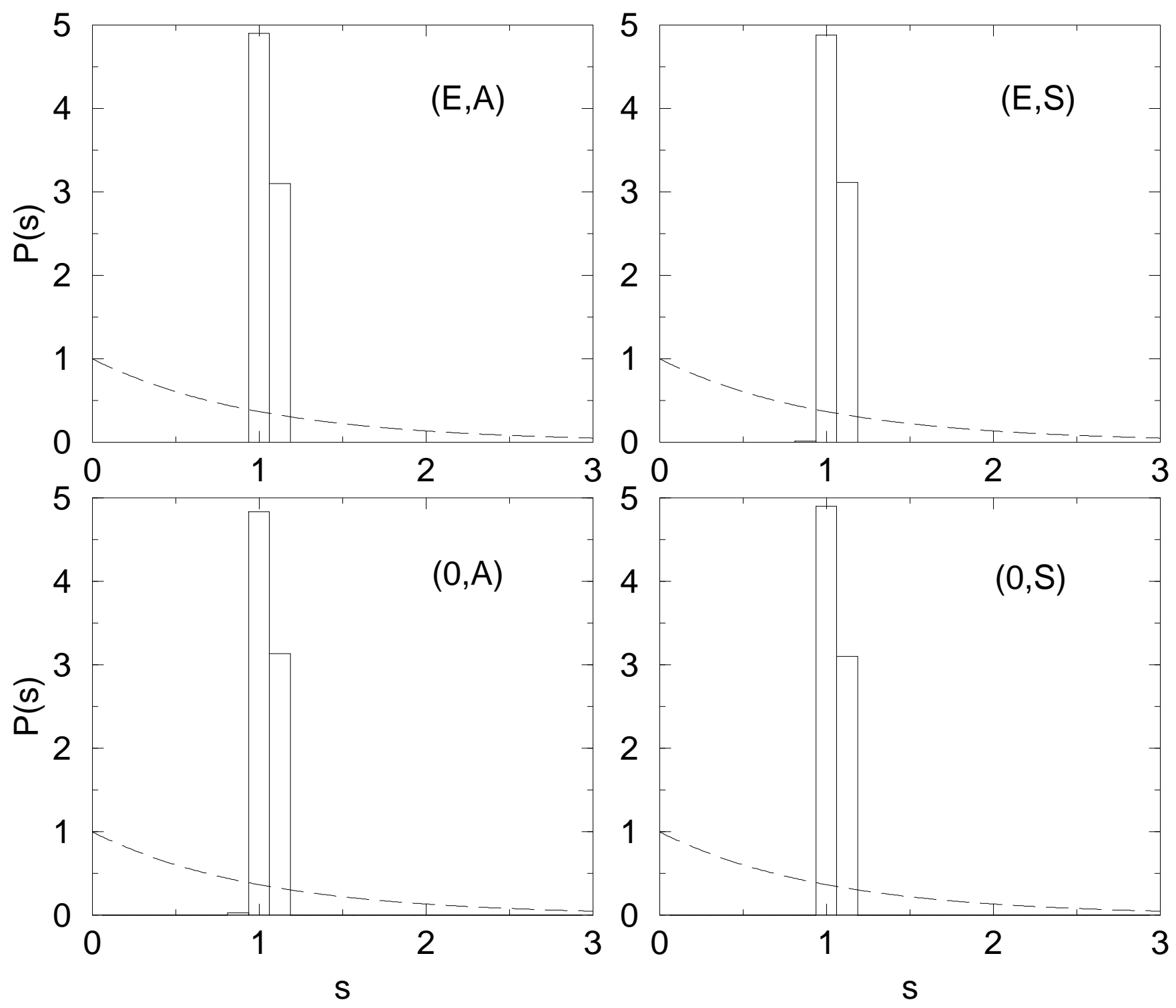

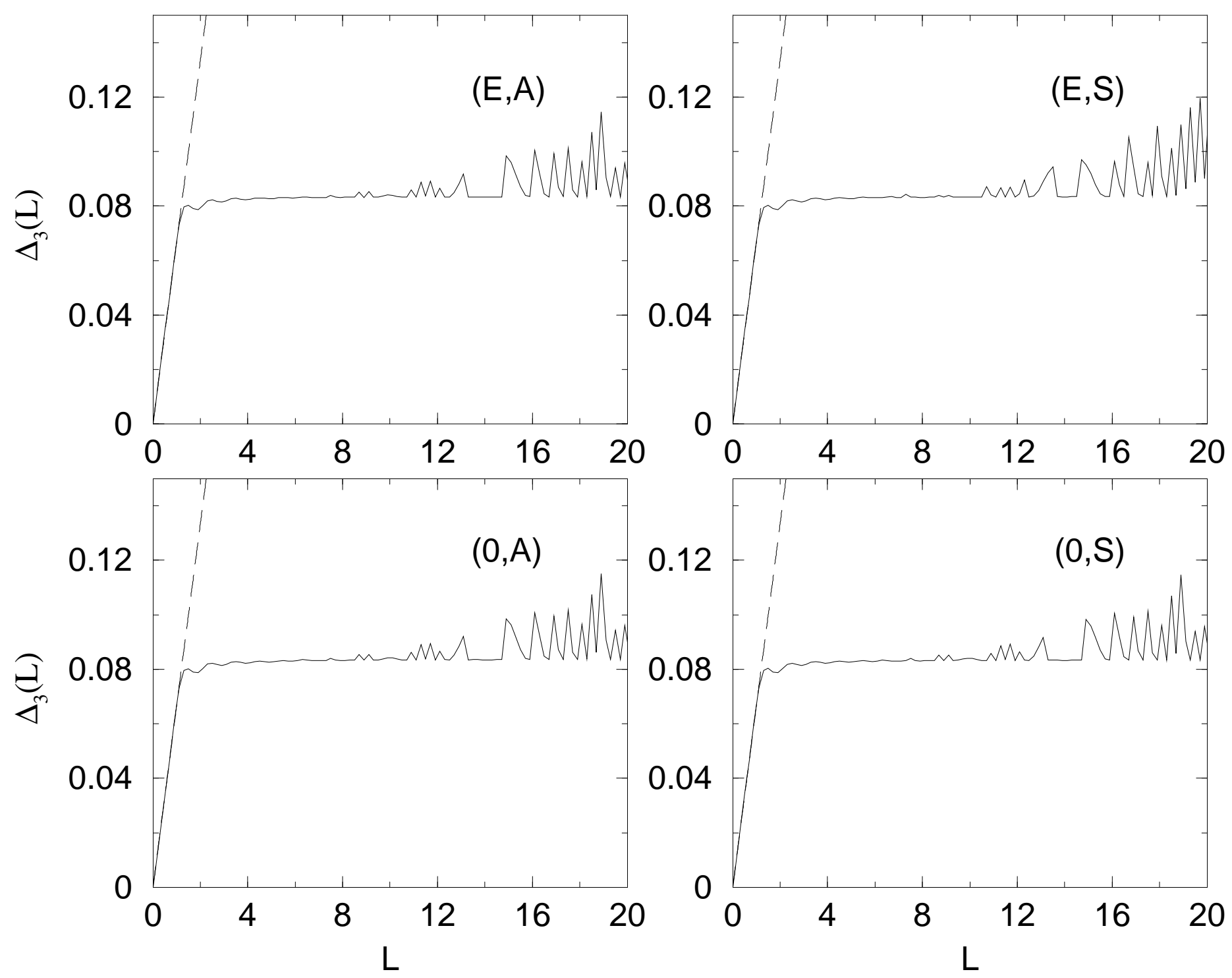

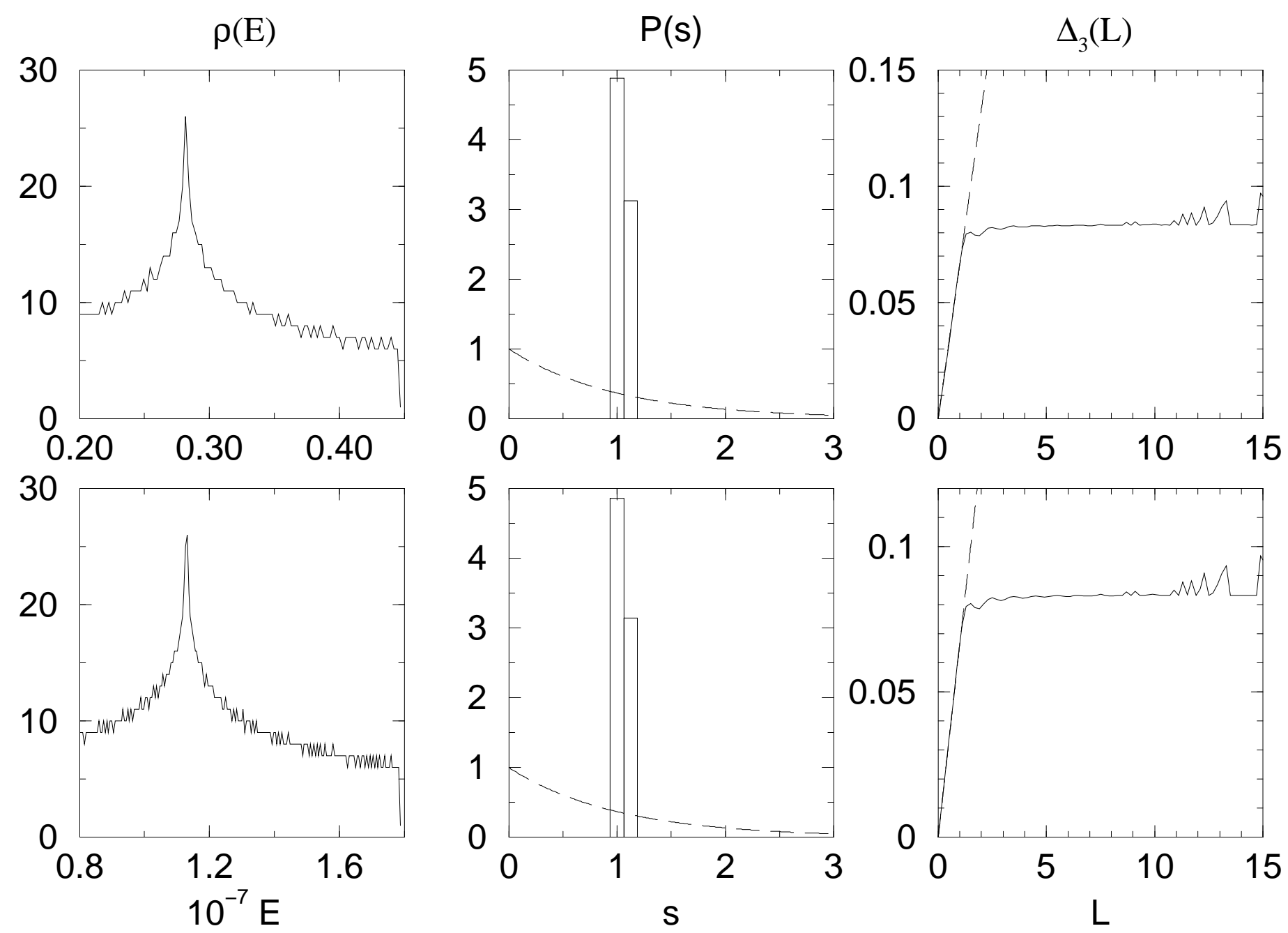


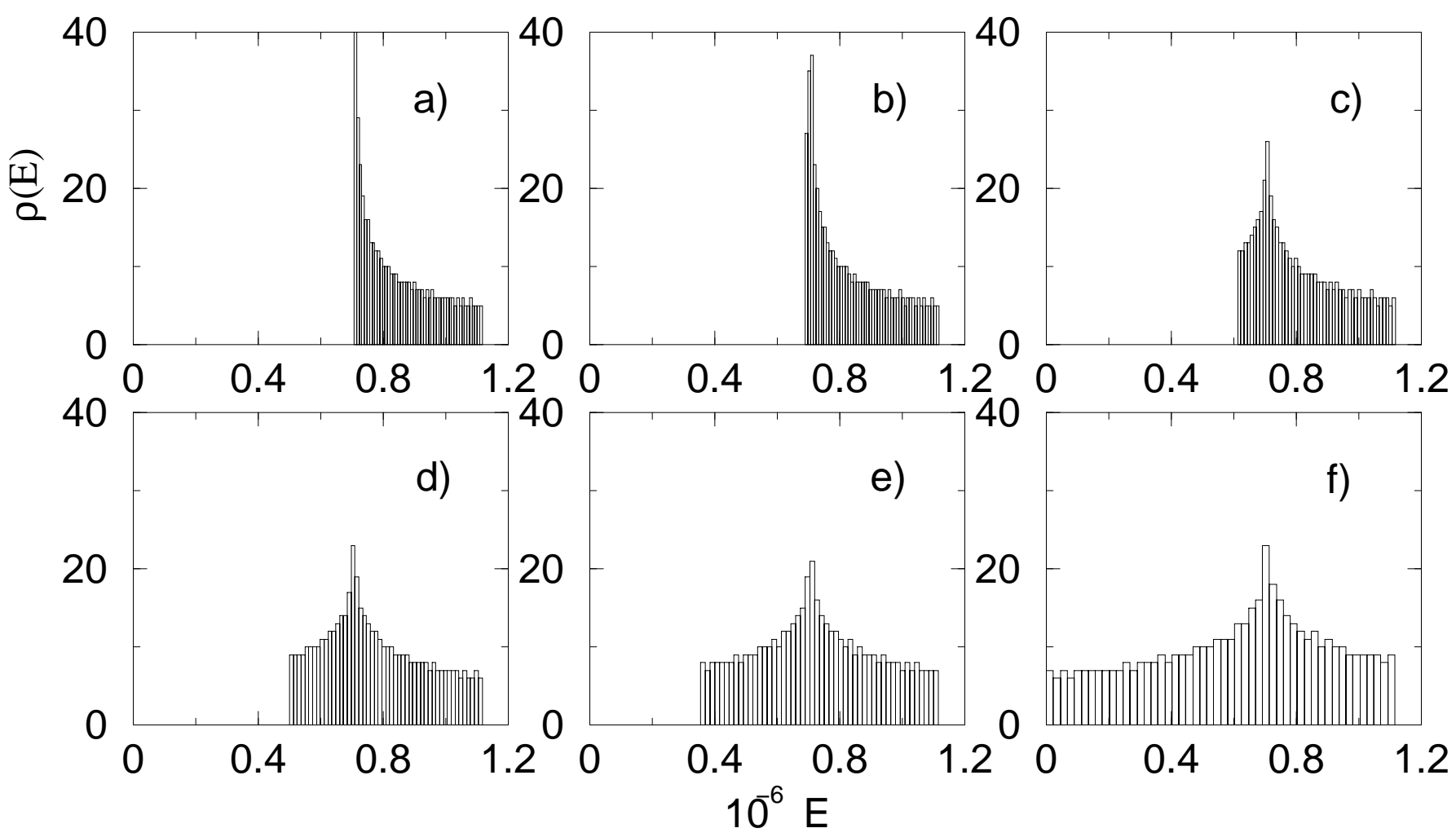




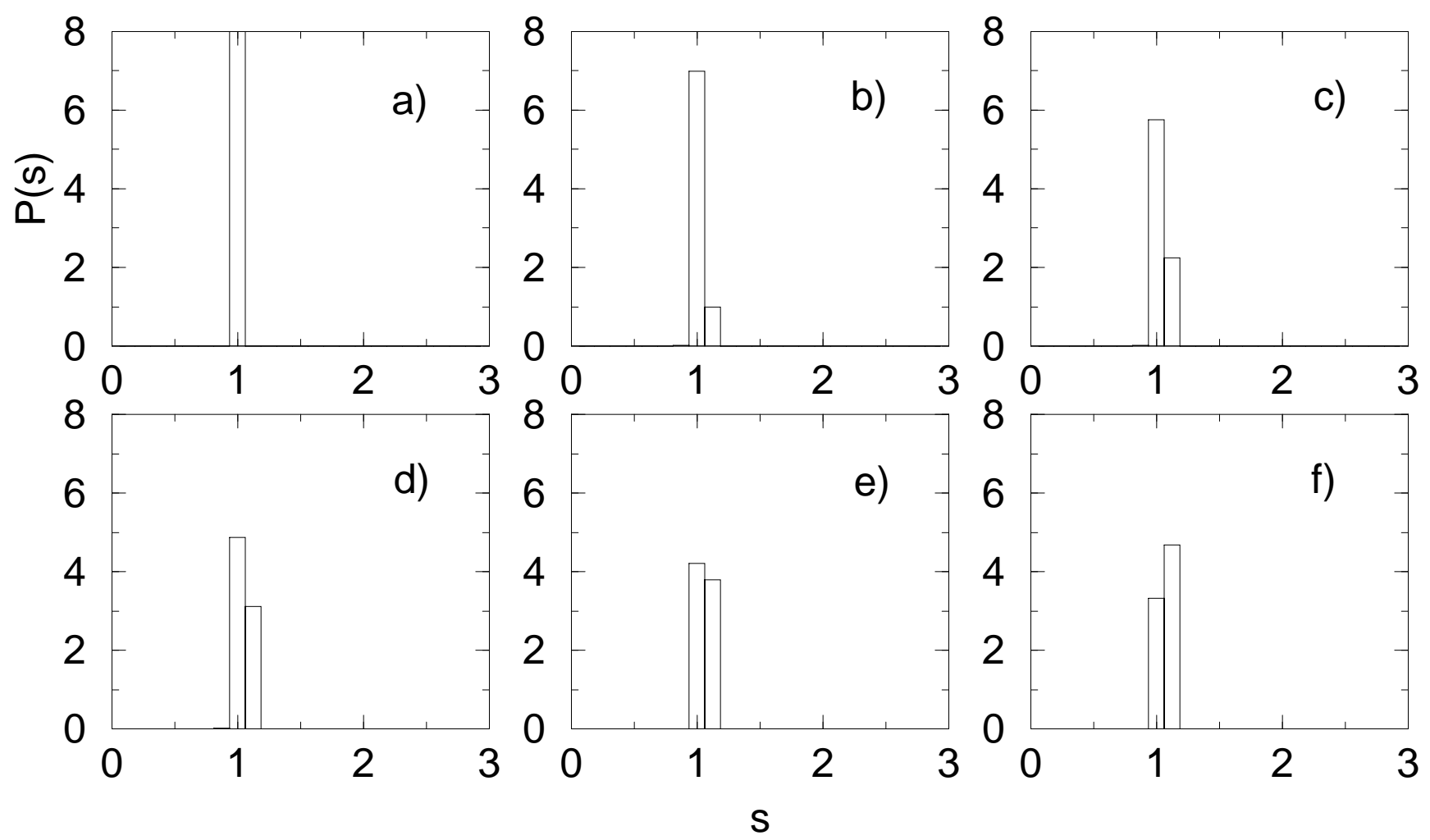




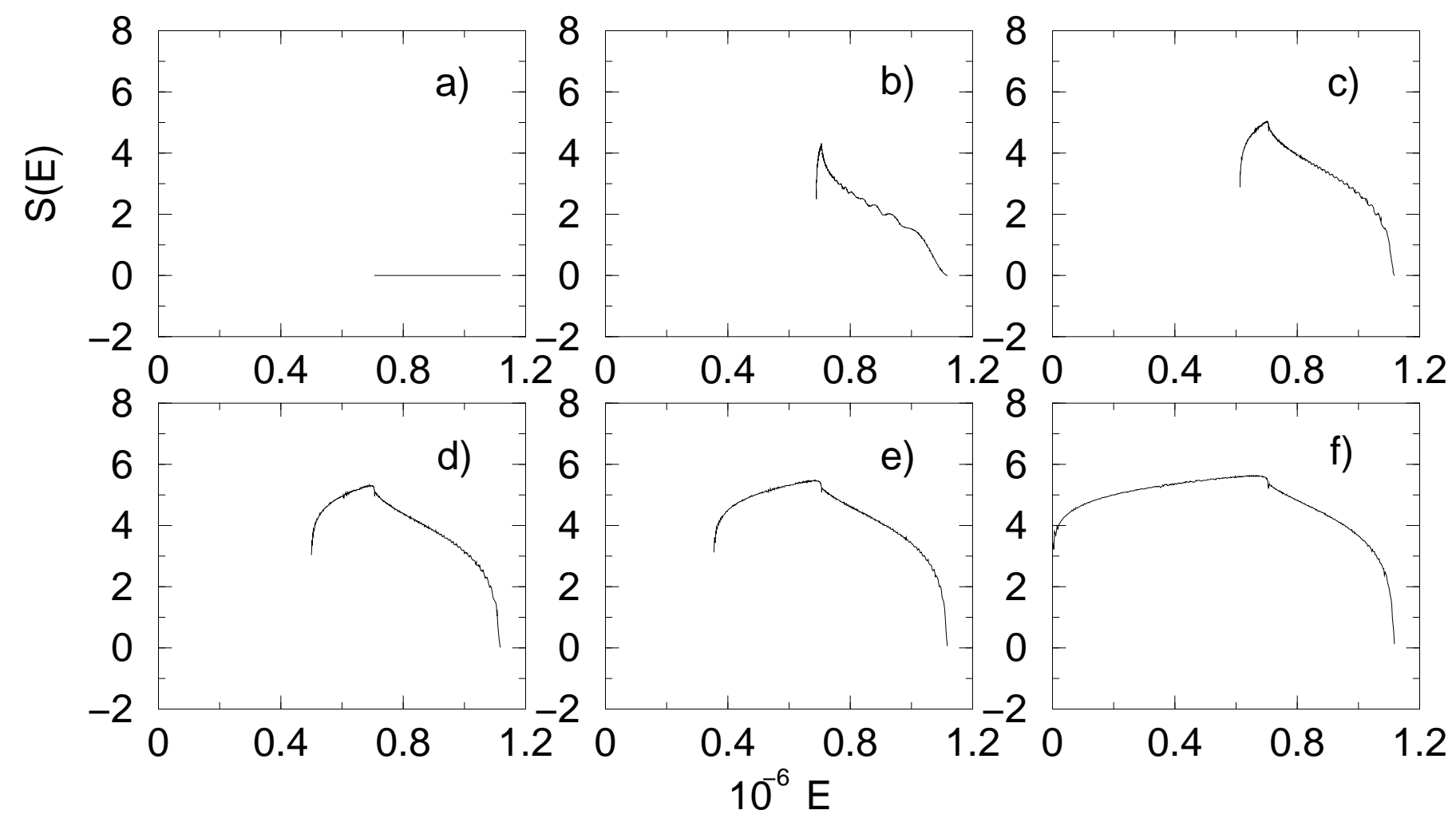

